\title{
Realization of Low RCS and Wide Beam Radiating Element for Ku- Band Electronically Steered Phased Array
}

\author{
Raj Kumar $^{1}$, Pramendra Kumar Verma ${ }^{1 *}$, M.V. Kartikeyan ${ }^{2}$ \\ ${ }^{1}$ Defence Electronics Application Laboratory, Dehradun, India \\ ${ }^{3}$ Indian Institute of Technology, Roorkee, India \\ *pramendra@deal.drdo.in
}

\begin{abstract}
The present paper discusses the design, analysis, and development of a low profile, low RCS, diagonally polarized and wide beam radiating element at Ku-Band for Electronically Steered Phased Array Antenna (ESPAA) for airborne applications. Radar Cross Section (RCS) of the antenna has been minimized by proper selection of substrate and eliminating the diffraction and scattering of the incident wave through the antenna. Finite element method (FEM) based full wave ANSYS's HFSS EM Simulation software was used for simulation and optimization of the antenna. The measured half power beam width of the antenna element is 90 degree and RCS better than - $30 \mathrm{dBsm}$ over the wide angular coverage of radiating element.
\end{abstract}

Keywords: Phased Array Antenna, RCS, SATCOM, VSWR

\section{Introduction}

An aircraft equipped with a satellite terminal has the capability to establish continuous satcom data link, during its movement. Presently, reflector based antennas mounted on the positioning system is being used for satellite communication which has larger volume and weight. Being a mechanical system, the beam steering is done mechanically hence its response time is more, requires periodic maintenance and the maintenance cost of these systems are quite high. By replacing the mechanical steering with electronic steering, the system can be made with quite a low volume and light in weight. Electronically steering antenna system also has the advantages of high reliability, low scan time and graceful degradation in case failures of some transmit/receive module.

Nowadays, electronically steered antennas have become the most preferred antenna for satellite communications. To minimize the scan loss during electronic steering of the beam, the radiating element of ESPAA should have wider 3-dB beamwidth. Microstrip based planar antenna has been preferred for airborne applications due to its lightweight, low profile and better integration with the aircraft. The microstrip antenna, being a resonant antenna has smaller bandwidth which can be improved by increasing the separation between the patch and ground plane by using thicker substrate. Unfortunately, this leads to the generation of a surface wave which further affects the mutual coupling and scattering behavior of the antenna system. The scattered fields from the antenna system also affect the RCS of the antenna. The bandwidth of microstrip antenna can be increased by several methods as discussed in the literature [1-8]. Using stacked patches or coplanar parasitic element are some of the ways to enhance the bandwidth of the microstrip antenna [4-6]. Number of techniques have been discussed in the literature [9-18] to reduce the RCS of a microstrip antenna array. However, none of the studies have considered the wideband radiating element having wide beamwidth required for large beam steering of ESPAA.
In the present paper, the design and development of a low RCS, diagonally polarized, wide beam radiating element for $\mathrm{Ku}$ band ESPAA is discussed. Diagonal polarization has been selected so that transmitted signals may be received by vertical as well as a horizontally polarized antenna. It also reduces the polarization mismatch losses during movement of the airborne platform. Half power beamwidth of the individual radiating element has been selected as 90 degrees to cater for beam steering of \pm 45 degree with $3 \mathrm{~dB}$ scan loss. The designed antenna has less than $-30 \mathrm{dBsm}$ radar crosssection over its beam coverage.

\section{Design, simulation, and results}

The phased array antenna consists of radiating elements followed by phase shifters, digital attenuators, and manifold networks. The resultant radiation pattern of phased array antenna is determined by the vector sum of the fields radiated by radiated elements including the effect of the digital phase shifter and digital attenuators. The performance of the radiating element decides not only the overall radiation pattern of the antenna but they also dictate the beam steering angle with specified scan loss. For airborne applications, low profile, lightweight microstrip antenna is the most suitable radiating element.

The parameters of the radiating element for ESPAA are completely different from that for the communication system. In the communication system, the radiating element should have larger gain and narrower beamwidth, but in case of ESPAA, the radiating element must have a broader beam so that large beam steering angle can be obtained [19]. In the case of airborne applications, the placement of the antenna element over the airborne platform should also not increases its resultant RCS.

A microstrip patch antenna has metallic radiating patch and ground plane separated by dielectric substrate material. Selection of dielectric material is done based on the beamwidth requirement of the antenna element. Dielectric materials with high dielectric constant reduce the size of the 
antenna element and hence increasing the beamwidth of the antenna [15]. The larger value of the dielectric constant of substrate material results in a reduction of antenna efficiency. Hence dielectric materials with high dielectric constant and low loss tangent is most suitable for radiating element. Further, the smaller radiating element would have lower RCS as compared to that of the larger radiating element. Considering the above facts, 60 mil TMM 4 material $\left(\varepsilon_{\mathrm{r}}=4.5\right.$, $\tan \delta=0.002$ ) has been selected in the design of radiating element. Size of radiating elements depends on the operating frequency and dielectric properties of the substrate material. Diagonally fed square patch antenna has been selected for excitation of the dual orthogonal mode of patch antenna simultaneously to generate the diagonally polarized wave. In this, the equal amplitude of orthogonal polarizations (both vertical and horizontal) is generated. Designed radiating element was modeled, simulated and optimized for the required beamwidth using ANSYS HFSS. Figure 1 shows the three dimensional CAD model of the radiating element.

The optimized size and feeding point of the radiating element are $4.35 \mathrm{~mm} \times 4.35 \mathrm{~mm}$ and at $(0.9 \mathrm{~mm}, 0.9 \mathrm{~mm})$ from the center of patch respectively. Comparison of beamwidth of the radiating element with RT duroid dielectric material ( $\mathrm{\varepsilon r}$ $=2.2)$ and TMM4 dielectric material $(\varepsilon r=4.5)$ is shown in Figure 2(a). It can be seen from the Figure 2a that using TMM4 as substrate material, the beamwidth of the antenna increases by 10 degree which results in \pm 5 degrees more beam steering as compared to that with RT Duroid 5880 substrate material.

RCS of the antenna is affected by scattering of the electromagnetic wave from the antenna. Increase in the scattering of EM wave increases the radar cross-section. RCS of the antenna element has been reduced by optimizing the scattering from all the edges of the antenna element and using high dielectric constant substrate to reduce the antenna element size. IE (Integral Equation) solver of HFSS has been used for the analysis of RCS of the antenna. The RCS of the antenna element with RT duroid dielectric material and TMM4 dielectric material is shown in Figure 2 (b).

It is clear from Figure $2 b$ that using TMM4, RCS of the antenna lowers by more than $15 \mathrm{~dB}$ at 90 -degree elevation angle. Figure 3 shows the photograph of the fabricated antenna. Developed radiating element was evaluated for its radiation pattern performance over Ku-band SATCOM transmit frequency band $(13.75 \mathrm{GHz}$ to $14.50 \mathrm{GHz})$.

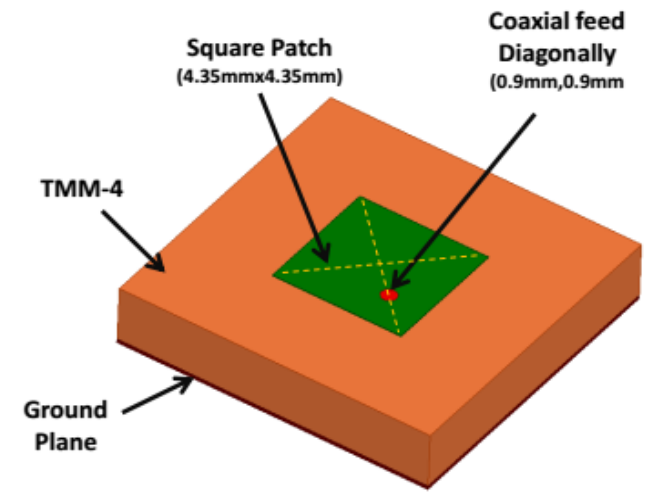

Fig. 1. 3D CAD model of the radiating element

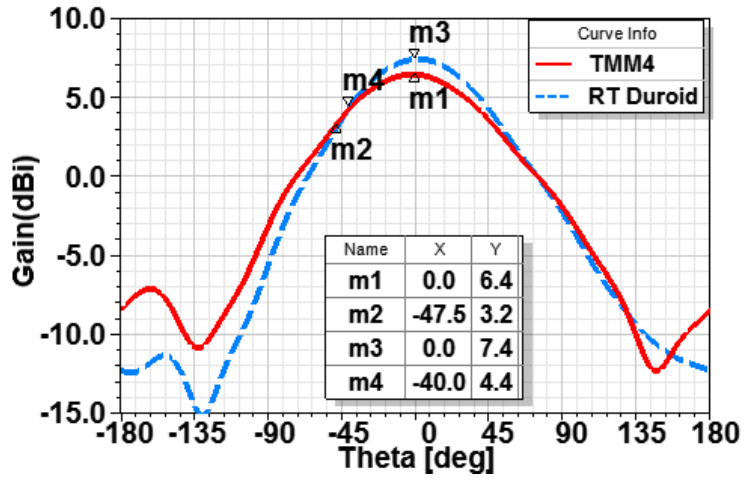

(a)

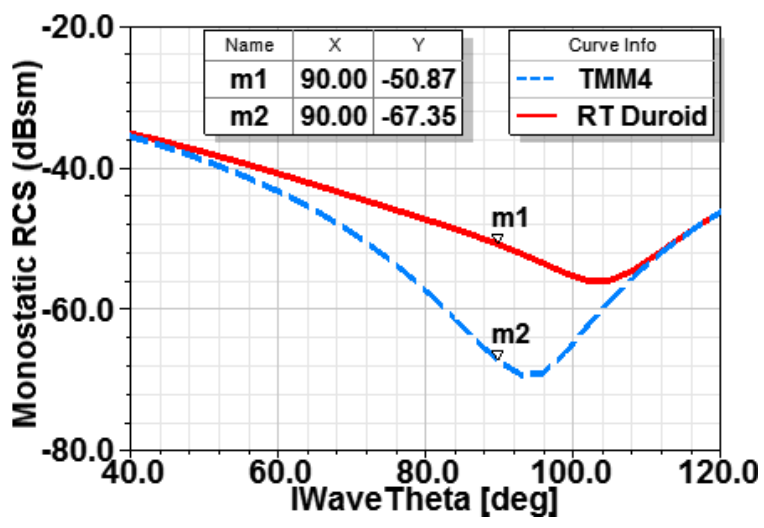

(b)

Fig. 2. Simulated Parameters with different dielectric material (a) Radiation pattern (b) RCS

Comparison of measured and simulated input reflection coefficient $\left(\mathrm{S}_{11}\right)$, E-plane and $\mathrm{H}$-plane radiation pattern of the antenna are shown in Figure 4 and Figures 5a, 5b respectively. Figure 6 shows measured RCS against the simulated RCS values. The antenna has the impedance bandwidth of $1.2 \mathrm{GHz}$ $(13.6$ to $14.8 \mathrm{GHz})$. The realized antenna has a $3 \mathrm{~dB}$ beamwidth of 90 degrees and RCS less than $<-30 \mathrm{dBsm}$. The measured radiation pattern of the radiating element follows the simulated pattern quite closely. RCS simulation was carried out using controlled (ideal) boundary conditions and also the fabrication tolerances were not considered, hence the simulation shows better RCS values. In practical measurement reflectivity of an anechoic chamber, the dynamic range of receiver and nearby reflections from positioning system affect the measured RCS. Due to these limitations, the measured RCS of the antenna is slightly less than the simulated values.

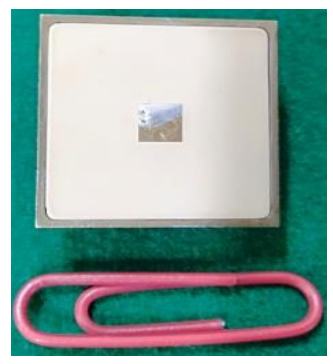

Fig. 3. Developed radiating element 


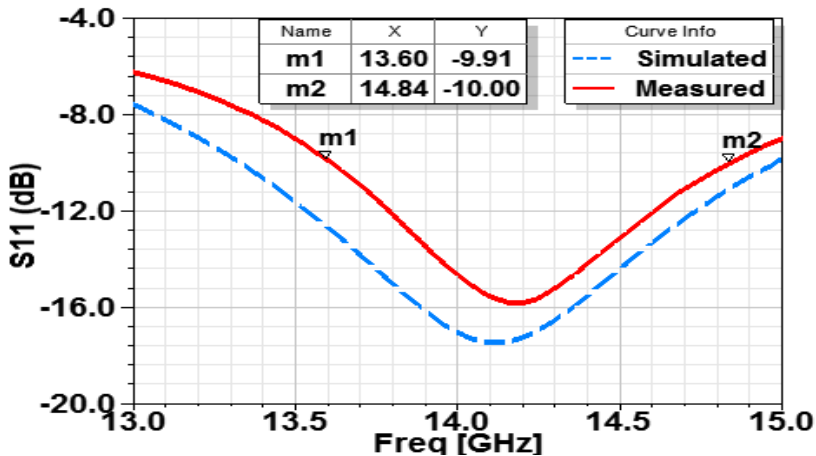

Fig. 4. S11 plot of radiating element

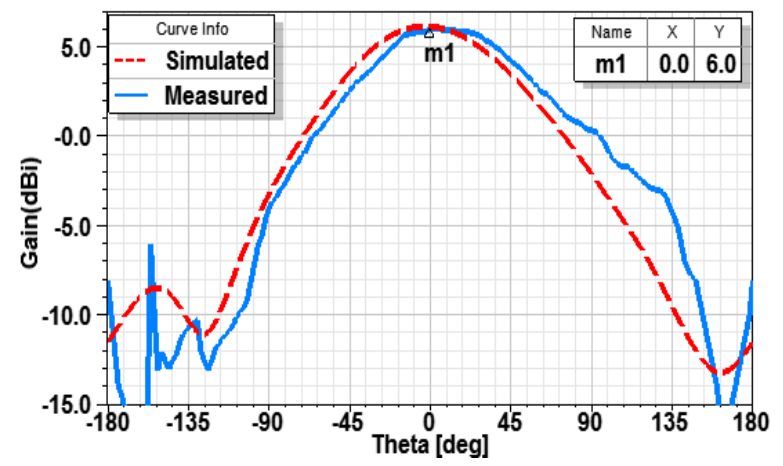

(a)

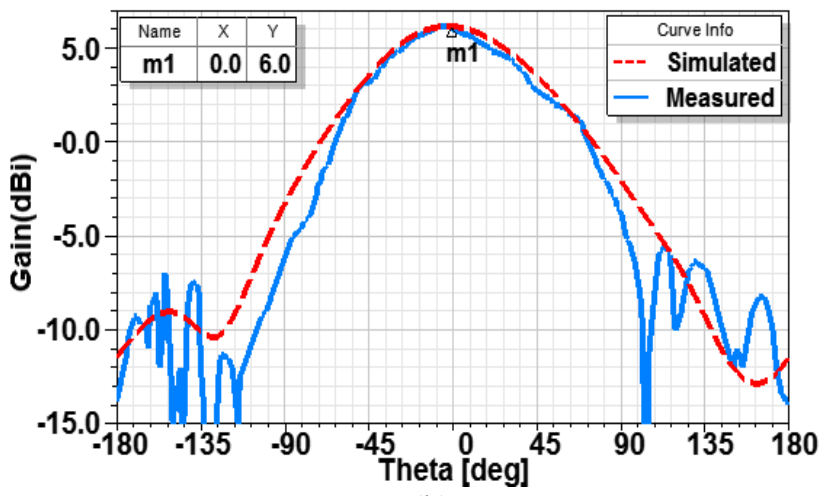

(b)

Fig. 5. Radiation pattern @ $14 \mathrm{GHz}$ of radiating element (a) E-plane (b) H-plane

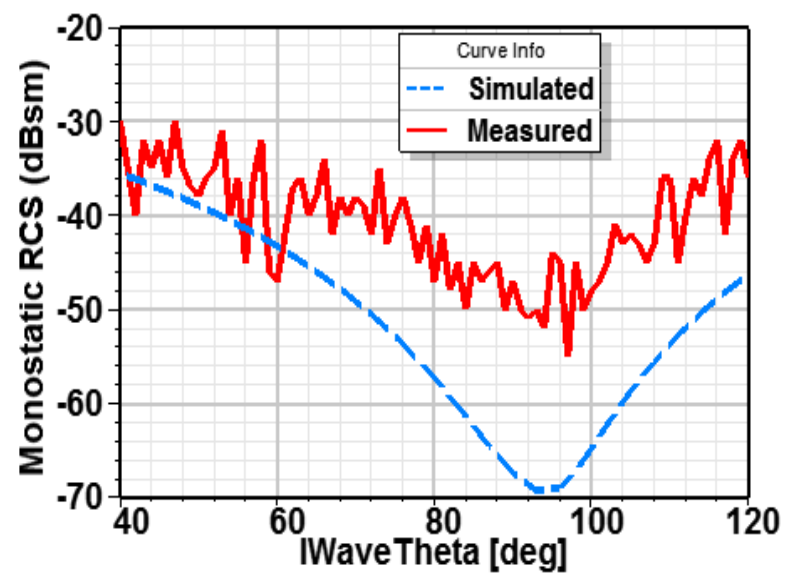

Fig. 6. RCS plot of radiating element

\section{Conclusion}

A low profile, low RCS, diagonally polarized and wide beam radiating element has been designed, developed and evaluated. The measured beamwidth of the antenna is 90 degree resulting in \pm 45 degrees beam steering with $3 \mathrm{~dB}$ scan loss. Due to wide beamwidth of the radiating element, it is best preferred radiating element for ESPAA with large beam steering. The designed radiating element has very low RCS (less than $-30 \mathrm{dBsm}$ ) which is a prime requirement for stealth platform. The developed antenna has lightweight, low profile and low RCS hence it is the most suitable radiating element for transmitting frequency band of $\mathrm{Ku}$ band SATCOM for ESPAA for airborne applications.

\section{Acknowledgment}

The authors are thankful to Director, Defence Electronics Applications Laboratory, Dehradun (Uttarakhand), India for granting permission to publish this paper.

\section{References}

[1] G. Kumar and K. P. Ray, Broadband Microstrip Patch Antennas, Artech House, pp. 151-169, 2003, ch. 4.

[2] J. R. James and P. S. Hall, Handbook of Microstrip Antennas, IET, 1989.

[3] Bhal I J and Bhartia P, Microstrip Antennas, Artech House, pp. $230-234,1980$.

[4] S. D. Targonski, R. B. Waterhouse and D. M. Pozar, Wideband aperture-coupled stacked patch antenna using thick substrate, Electron. Letters, vol. 32, no. 21, pp. 1941-1942, 1996.

[5] A. Serra, P. Nepa, G. Manara, G. Tribellini and S. Cioci, A wideband dual-polarized stacked patch antenna, IEEE Antennas and Wireless Propagation Letters, vol. 6, pp. 141-143, 2007.

[6] M. M. Bilgic and K. Yegin, Wideband high-gain aperture coupled antenna for $\mathrm{Ku}$ band phased array antenna systems, Microwave and Optical Technology Letters, vol. 55, no. 6, pp. 1291-1295, 2013.

[7] S. L. Mallikarjun, R.G. Madhuri, S. A. Malipatil, and P. M. Hadalgi, Development of microstrip array antenna for wide band and multi-band applications, Indian Journal of Radio \& Space Physics, vol. 38, pp. 289-294, 2009.

[8] P. M. Hadalgi and S. L. Mallikarjun, Design and development of microstrip array antenna with broader bandwidth and beam, Int. Journal of Electronics Engineering, vol. 1, no. 2, pp. 251-253, 2009.

[9] Jackson D.R., A multiple-layer radome for reducing the RCS of microstrip patch, In Proceeding IEEE International Symposium Digest on Ant. Prop., Dallas (USA), pp. 374-377, 1990.

[10] R. S. Chu, Analysis of an infinite phased array of dipole elements with RAM coating on ground plane and covered with layered radome, IEEE Transaction on Antennas and Propagation., vol39, pp. 164-176, 1991.

[11] D. M. Pozar, RCS reduction for a microstrip antenna using a normally biased ferrite substrate, IEEE Microwave and Guided Wave Letters, vol. 2, pp. 196-198, 1992.

[12] M. Gustafsson, RCS reduction of integrated antenna arrays with resistive sheets, Journal of Electromagnetic Waves and Applications, vol. 20, no.1, pp 3479-3482, 2006.

[13] S. C. Zhao, B. Z, Wang, and Q. Q. He, Broadband radar cross section reduction of a rectangular patch antenna, Progress In Electromagnetics Research, vol. 79, pp. 263-275, 2008.

[14] A. N. Lagarkov, V. N. Kisel, and V. N. Semenenko, Wide angle absorption by the use of a meta-material plate, Progress In Electromagnetics Research Letters, vol. 1, pp. 35-44, 2008.

[15] D. Singh and V. M. Srivastava, An analysis of RCS for dual-band slotted patch antenna with a thin dielectric using shorted stubs metamaterial absorber, International Journal of Electronics and Communications, vol. 90, pp. 53-62, 2018. 
[16] T. A. Khan, J. Li, Z. Li, M. Abdullah, and A. Zhang, Design of a Vivaldi antenna with wideband reduced radar cross-section, International Journal of Electronics and Communications, vol. 95, pp. 47-51, 2018

[17] Mahsa Ahmadi Rad, Mohammad Reza Soheilifar and Ferdows B. Zarrabi, Compact microstrip antenna based on fractal metasurface with low radar cross section and wide bandwidth, International Journal of Electronics and Communications, vol. 98, pp. 74-79, 2019.

[18] A. Sharma, D. Gangwar, B. K. Kanaujia, and S. Dwari, RCS reduction and gain enhancement of SRR inspired circularly polarized slot antenna using metasurface, AEU-International Journal of Electronics and Communications, vol. 91, pp. 132 142,2018

[19] Kumar and M. V. Kartikeyan, On the selection of radiating elements for electronically steered phased array antenna for SATCOM applications", $14^{\text {th }}$ IEEE India Council International conference (INDICON), 2017.

\section{Biography of the author}

Raj Kumar obtained his M. E. degree in Microwave \& Radar from the University of Roorkee(presently IIT Roorkee). He has published more than thirty papers in International journals and IEEE conferences. His areas of interest include microwave and mm-wave antennas design including omnidirectional antenna, fan beam antenna, slotted array antenna, antenna for radiometry, microstrip antennas, design of waveguide filters, reflector antennas, horn antennas, conformal antennas, phased array antenna, etc. He has been awarded the DRDO Technology Award, Group Technology award, \& Laboratory Scientist of the Year award. He is a fellow of the ATMS Society.

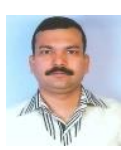

Pramendra Kumar Verma received his B. Tech degree in Electronics Engineering from V.B.S.P.U., Jaunpur and M.E. degree in Microwave Engineering from J.E.C., Jabalpur. He has published numbers of paper in International journals and IEEE conferences.

His area of research includes the design and development of microwave and mmwave antennas, slotted waveguide omnidirectional antenna, conformal phased array antenna, reflector antenna, waveguide components, etc. $\mathrm{He}$ is a recipient of Laboratory Scientist of the Year Award 2015. He is also a reviewer of International Journals IJMWT, EuMA USA \& IJAMT, India. He is a member of ATMS society and a life member of IETE.

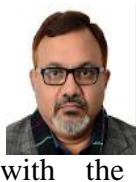

M.V. Kartikeyan received the M.Sc. degree in physics, and the Ph.D. degree in Electronics Engineering from IIT (BHU) Varanasi, India, in 1985 and 1992, respectively. He has been a Full Professor with the Department of Electronics and Communication Engineering, IIT Roorkee, Roorkee, India, since 2009. His current research interests include millimeter/ terahertz wave engineering. 